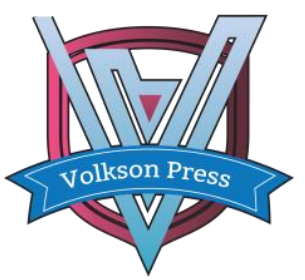

\title{
The Influence of FDI on the Spatial and Temporal Differentiation of the Maturity of Energy Saving and Carbon Emission Reduction in Manufacturing Industry -- a Case Study of Chinese Central and Eastern Regions
}

\author{
Yang Feixue ${ }^{a}$
}

aDepartment of Economics, Liaoning University of Technology, ShiyingStreet, JinzhouCity, China

This is an open access article distributed under the Creative Commons Attribution License, which permits unrestricted use, distribution, and reproduction in any medium, provided the original work is properly cited

\section{ARTICLE DETAILS}

\section{Article History:}

Received 02 october 2017 Accepted 06 october 2017 Available online 11 october 2017

Keywords:

FDI; manufacturing industry; the maturity of energy saving and carbon emission reduction; spatial and temporal differentiation

\section{ABSTRACT}

This paper first introduces the manufacturing maturity status of energy saving and carbon emission reduction, and then selects the data of 19 provinces in eastern and central regions of China from 2003 to 2012. And the mathematical statistical model and set pair analysis method based on fuzzy rules are used to do the research on the influence of FDI on the spatial and temporal differentiation of the maturity of energy saving and carbon emission reduction in manufacturing industry. The empirical analysis shows that, in terms of time, the impact of FDI on the maturity of energy saving and carbon emission reduction in manufacturing industry in 2012 was higher than that in 2003 in Chinese central and eastern regions. In terms of space, the impact of FDI in the eastern region on the maturity of energy saving and carbon emission reduction in the manufacturing sector is significantly higher than that in the central region. Finally, some suggestions are put forward to improve the maturity of energy saving and carbon emission reduction in manufacturing enterprises from the aspects of enterprises, Chinese government and regions.

\section{Introduction}

In recent years, the "haze" phenomenon in major cities is constantly serious. Chinese government has been highly aware of the serious problems of the environment, and put forward the target of reducing carbon emissions. Manufacturing industry is an important industry in China and one of the economic lifelines of the country. However, in the central and eastern regions of China, the distribution of manufacturing industry is uneven, the utilization rate of energy consumption is different, and the pollution control is also different. This paper analyzes the impact of FDI on the spatial and temporal differentiation of the maturity of the energy saving and emission reduction in China's central and eastern manufacturing sector, and hopes to provide reasonable suggestions for the energy saving and emission reduction and sustainable economic development of Chinese manufacturing enterprises.

2. The Maturity Status of Energy Saving and Carbon Emission Reduction of Manufacturing Industry in East and Central Regions

The maturity of energy saving and carbon emission reduction in manufacturing industry refers to the degree of energy saving and carbon emission reduction, indicating the developing level of energy saving and emission reduction in manufacturing industry. Table 1 shows the relative maturity indexes and stages of the energy saving and carbon emission reduction of provinces in central and eastern China in 2003 and 2012. Relative to 2003, three relative maturity indexes of provinces in 2012 generally promoted in different amplitude. Among them, the average relative development index of provinces is significantly higher than the average relative coordination index. Under the combined effect of relative development index and relative coordination index, the maturity indexes of 3 provinces are at a very high level, and the maturity indexes of 16 provinces are at a higher level in 2012.

Table1. Relative Maturity Indexes and Phases of Energy Saving and Carbon Emission Reduction of Central and Eastern Manufacturing Industry

\begin{tabular}{|c|c|c|c|c|c|c|c|c|c|c|c|c|c|}
\hline $\begin{array}{l}\mathrm{r} \\
\mathrm{e}\end{array}$ & \multirow{3}{*}{$\begin{array}{l}\text { provi } \\
\text { nces }\end{array}$} & \multicolumn{4}{|c|}{$\begin{array}{l}\text { development } \\
\text { index }\end{array}$} & \multicolumn{4}{|c|}{$\begin{array}{l}\text { coordination } \\
\text { index }\end{array}$} & \multicolumn{4}{|c|}{$\begin{array}{l}\text { coordinated } \\
\text { development } \\
\text { index }\end{array}$} \\
\hline g & & \multicolumn{2}{|c|}{2003} & \multicolumn{2}{|c|}{2012} & \multicolumn{2}{|c|}{2003} & \multicolumn{2}{|c|}{2012} & \multicolumn{2}{|c|}{2003} & \multicolumn{2}{|c|}{2012} \\
\hline $\begin{array}{l}\text { l } \\
\text { o } \\
\text { n } \\
\text { s }\end{array}$ & & $\begin{array}{l}\text { in } \\
d \\
\text { e } \\
x\end{array}$ & $\begin{array}{l}\mathrm{s} \\
\mathrm{t} \\
\mathrm{a} \\
\mathrm{g} \\
\mathrm{e}\end{array}$ & $\begin{array}{l}\text { in } \\
\text { de } \\
x\end{array}$ & $\begin{array}{l}\mathrm{s} \\
\mathrm{t} \\
\mathrm{a} \\
\mathrm{g} \\
\mathrm{e}\end{array}$ & $\begin{array}{l}\text { in } \\
d \\
e \\
x\end{array}$ & $\begin{array}{l}\mathrm{s} \\
\mathrm{t} \\
\mathrm{a} \\
\mathrm{g} \\
\mathrm{e}\end{array}$ & $\begin{array}{l}\text { in } \\
d \\
\text { e } \\
x\end{array}$ & $\begin{array}{l}\text { st } \\
\text { ag } \\
\text { e }\end{array}$ & $\begin{array}{l}\text { in } \\
d \\
e \\
x\end{array}$ & $\begin{array}{l}\mathrm{s} \\
\mathrm{t} \\
\mathrm{a} \\
\mathrm{g} \\
\mathrm{e}\end{array}$ & in & $\begin{array}{l}\mathrm{s} \\
\mathrm{t} \\
\mathrm{a} \\
\mathrm{g} \\
\mathrm{e}\end{array}$ \\
\hline & $\begin{array}{l}\text { Beijin } \\
\mathrm{g}\end{array}$ & $\begin{array}{l}9 \\
0 . \\
9 \\
7\end{array}$ & $\begin{array}{l}\text { I } \\
\text { V }\end{array}$ & $\begin{array}{l}10 \\
0 . \\
00\end{array}$ & $\begin{array}{l}\text { I } \\
\text { V }\end{array}$ & $\begin{array}{l}7 \\
4 . \\
4 \\
7\end{array}$ & $\begin{array}{l}\text { II } \\
\text { I }\end{array}$ & $\begin{array}{l}7 \\
9 . \\
6\end{array}$ & III & $\begin{array}{l}8 \\
2 . \\
3 \\
1\end{array}$ & $\begin{array}{l}\text { II } \\
\text { I }\end{array}$ & $\begin{array}{l}8 \\
9 . \\
2 \\
2\end{array}$ & $\begin{array}{l}\text { I } \\
\text { V }\end{array}$ \\
\hline $\begin{array}{l}E \\
a\end{array}$ & $\begin{array}{l}\text { Tianji } \\
\mathrm{n}\end{array}$ & $\begin{array}{l}9 \\
4 . \\
1 \\
3\end{array}$ & $\begin{array}{l}\text { I } \\
\text { V }\end{array}$ & $\begin{array}{l}95 \\
.5 \\
6\end{array}$ & $\begin{array}{l}\text { I } \\
\text { V }\end{array}$ & $\begin{array}{l}7 \\
8 . \\
3 \\
7\end{array}$ & $\begin{array}{l}\text { II } \\
\text { I }\end{array}$ & $\begin{array}{l}6 \\
8 . \\
9 \\
7\end{array}$ & II & $\begin{array}{l}8 \\
5 . \\
8 \\
9\end{array}$ & $\begin{array}{l}\text { I } \\
\text { V }\end{array}$ & $\begin{array}{l}8 \\
1 . \\
1 \\
8\end{array}$ & $\begin{array}{l}\text { II } \\
\text { I }\end{array}$ \\
\hline $\begin{array}{l}\mathrm{s} \\
\mathrm{t} \\
\mathrm{e} \\
\mathrm{r}\end{array}$ & Hebei & $\begin{array}{l}6 \\
2 . \\
7 \\
1\end{array}$ & II & $\begin{array}{l}84 \\
.2 \\
9\end{array}$ & $\begin{array}{l}\text { II } \\
\text { I }\end{array}$ & $\begin{array}{l}6 \\
0 . \\
9 \\
3\end{array}$ & II & $\begin{array}{l}6 \\
5 . \\
3\end{array}$ & II & $\begin{array}{l}6 \\
1 . \\
8 \\
1\end{array}$ & II & $\begin{array}{l}7 \\
4 . \\
1 \\
9\end{array}$ & $\begin{array}{l}\text { II } \\
\text { I }\end{array}$ \\
\hline $\begin{array}{l}\mathrm{n} \\
\mathrm{r} \\
\mathrm{e} \\
\mathrm{g}\end{array}$ & $\begin{array}{l}\text { Liaon } \\
\text { ing }\end{array}$ & $\begin{array}{l}7 \\
4 . \\
2 \\
3\end{array}$ & $\begin{array}{l}\text { II } \\
\text { I }\end{array}$ & $\begin{array}{l}92 \\
.2 \\
6\end{array}$ & $\begin{array}{l}\text { I } \\
\text { V }\end{array}$ & $\begin{array}{l}6 \\
5 . \\
4 \\
6\end{array}$ & II & $\begin{array}{l}7 \\
1 . \\
6 \\
6\end{array}$ & III & $\begin{array}{l}6 \\
9 . \\
7\end{array}$ & II & $\begin{array}{l}8 \\
1 . \\
1 \\
3\end{array}$ & $\begin{array}{l}\text { II } \\
\text { I }\end{array}$ \\
\hline $\begin{array}{l}\mathrm{O} \\
\mathrm{n}\end{array}$ & $\begin{array}{l}\text { Shan } \\
\text { ghai }\end{array}$ & $\begin{array}{l}9 \\
7 . \\
2 \\
5\end{array}$ & $\begin{array}{l}\text { I } \\
\text { V }\end{array}$ & $\begin{array}{l}97 \\
.9 \\
6\end{array}$ & $\begin{array}{l}\text { I } \\
\text { V }\end{array}$ & $\begin{array}{l}7 \\
3 . \\
0 \\
7\end{array}$ & $\begin{array}{l}\text { II } \\
\text { I }\end{array}$ & $\begin{array}{l}7 \\
3 . \\
5 \\
2\end{array}$ & III & $\begin{array}{l}8 \\
4 . \\
3\end{array}$ & $\begin{array}{l}\text { II } \\
\text { I }\end{array}$ & $\begin{array}{l}8 \\
4 . \\
8 \\
7\end{array}$ & $\begin{array}{l}\text { II } \\
\text { I }\end{array}$ \\
\hline & $\begin{array}{l}\text { Jiang } \\
\text { su }\end{array}$ & $\begin{array}{l}9 \\
4 . \\
9 \\
9\end{array}$ & $\begin{array}{l}\text { I } \\
\text { V }\end{array}$ & $\begin{array}{l}97 \\
.1 \\
5\end{array}$ & $\begin{array}{l}\text { I } \\
\text { V }\end{array}$ & $\begin{array}{l}6 \\
6 . \\
9 \\
2\end{array}$ & II & $\begin{array}{l}6 \\
8 . \\
6 \\
3\end{array}$ & II & $\begin{array}{l}7 \\
9 . \\
7 \\
3\end{array}$ & $\begin{array}{l}\text { II } \\
\text { I }\end{array}$ & $\begin{array}{l}8 \\
1 . \\
6 \\
5\end{array}$ & $\begin{array}{l}\text { II } \\
\text { I }\end{array}$ \\
\hline
\end{tabular}




\begin{tabular}{|c|c|c|c|c|c|c|c|c|c|c|c|c|}
\hline $\begin{array}{l}\text { Zheji } \\
\text { ang }\end{array}$ & $\begin{array}{l}9 \\
6 . \\
6\end{array}$ & $\begin{array}{l}\mathrm{I} \\
\mathrm{V}\end{array}$ & $\begin{array}{l}96 \\
.4 \\
3\end{array}$ & $\begin{array}{l}\text { II } \\
\text { I }\end{array}$ & $\begin{array}{l}7 \\
3 . \\
1 \\
3\end{array}$ & $\begin{array}{l}\text { II } \\
\text { I }\end{array}$ & $\begin{array}{l}7 \\
0 . \\
2 \\
5\end{array}$ & III & $\begin{array}{l}8 \\
4 . \\
0 \\
5\end{array}$ & $\begin{array}{l}\text { II } \\
\text { I }\end{array}$ & $\begin{array}{l}8 \\
2 . \\
3 \\
3 \\
\end{array}$ & $\begin{array}{l}\text { II } \\
\text { I }\end{array}$ \\
\hline $\begin{array}{l}\text { Fujia } \\
\mathrm{n}\end{array}$ & $\begin{array}{l}9 \\
4 . \\
6 \\
4 \\
\end{array}$ & $\begin{array}{l}\mathrm{I} \\
\mathrm{V}\end{array}$ & $\begin{array}{l}95 \\
.7 \\
3\end{array}$ & $\begin{array}{l}\mathrm{I} \\
\mathrm{V}\end{array}$ & $\begin{array}{l}7 \\
4 . \\
4\end{array}$ & $\begin{array}{l}\text { II } \\
\text { I }\end{array}$ & $\begin{array}{l}7 \\
5 . \\
0 \\
4\end{array}$ & III & $\begin{array}{l}8 \\
3 . \\
9 \\
1\end{array}$ & $\begin{array}{l}\text { II } \\
\text { I }\end{array}$ & $\begin{array}{l}8 \\
4 . \\
7 \\
5\end{array}$ & $\begin{array}{l}\text { II } \\
\text { I }\end{array}$ \\
\hline $\begin{array}{l}\text { Shan } \\
\text { dong }\end{array}$ & $\begin{array}{l}8 \\
5 . \\
6 \\
3 \\
\end{array}$ & $\begin{array}{l}\mathrm{I} \\
\mathrm{V}\end{array}$ & $\begin{array}{l}94 \\
.7 \\
3\end{array}$ & $\begin{array}{l}\text { I } \\
\text { V }\end{array}$ & $\begin{array}{l}6 \\
3 . \\
6 \\
3 \\
\end{array}$ & II & $\begin{array}{l}6 \\
9 . \\
2 \\
8 \\
\end{array}$ & II & $\begin{array}{l}7 \\
3 . \\
8 \\
1 \\
\end{array}$ & $\begin{array}{l}\text { II } \\
\text { I }\end{array}$ & $\begin{array}{l}8 \\
1 . \\
0 \\
1\end{array}$ & $\begin{array}{l}\text { II } \\
\text { I }\end{array}$ \\
\hline $\begin{array}{l}\text { Guan } \\
\text { gdon } \\
\mathrm{g}\end{array}$ & $\begin{array}{l}1 \\
0 \\
0\end{array}$ & $\begin{array}{l}\mathrm{I} \\
\mathrm{V}\end{array}$ & $\begin{array}{l}97 \\
.8\end{array}$ & $\begin{array}{l}\mathrm{I} \\
\mathrm{V}\end{array}$ & $\begin{array}{l}7 \\
8 . \\
6 \\
4 \\
\end{array}$ & $\begin{array}{l}\text { II } \\
\text { I }\end{array}$ & $\begin{array}{l}7 \\
4 . \\
1 \\
6 \\
\end{array}$ & III & $\begin{array}{l}8 \\
8 . \\
6 \\
8 \\
\end{array}$ & $\begin{array}{l}\text { I } \\
\text { V }\end{array}$ & $\begin{array}{l}8 \\
5 . \\
1 \\
6 \\
\end{array}$ & $\begin{array}{l}\mathrm{I} \\
\mathrm{V}\end{array}$ \\
\hline $\begin{array}{l}\text { Haina } \\
\mathrm{n}\end{array}$ & $\begin{array}{l}7 \\
7 . \\
8 \\
8 \\
\end{array}$ & $\begin{array}{l}\text { II } \\
\text { I }\end{array}$ & $\begin{array}{l}82 \\
.4 \\
8\end{array}$ & $\begin{array}{l}\text { I } \\
\text { V }\end{array}$ & $\begin{array}{l}8 \\
4 . \\
2\end{array}$ & $\begin{array}{l}\text { II } \\
\text { I }\end{array}$ & $\begin{array}{l}7 \\
3 . \\
3 \\
2 \\
\end{array}$ & III & $\begin{array}{l}8 \\
0 . \\
9 \\
8 \\
\end{array}$ & $\begin{array}{l}\text { II } \\
\text { I }\end{array}$ & $\begin{array}{l}8 \\
2 . \\
3 \\
4\end{array}$ & $\begin{array}{l}\text { II } \\
\text { I }\end{array}$ \\
\hline $\begin{array}{l}\text { Shan } \\
\text { xi }\end{array}$ & $\begin{array}{l}4 \\
8 . \\
1 \\
3\end{array}$ & II & $\begin{array}{l}82 \\
.0 \\
3\end{array}$ & $\begin{array}{l}\text { II } \\
\text { I }\end{array}$ & $\begin{array}{l}5 \\
3 . \\
6 \\
4\end{array}$ & II & $\begin{array}{l}6 \\
5 . \\
3 \\
6\end{array}$ & II & $\begin{array}{l}5 \\
0 . \\
8 \\
1\end{array}$ & II & $\begin{array}{l}7 \\
3 . \\
2 \\
2\end{array}$ & $\begin{array}{l}\text { II } \\
\text { I }\end{array}$ \\
\hline Jilin & $\begin{array}{l}7 \\
3 . \\
9 \\
7 \\
\end{array}$ & \begin{tabular}{|l} 
II \\
I
\end{tabular} & $\begin{array}{l}90 \\
.7 \\
5\end{array}$ & $\begin{array}{l}\mathrm{I} \\
\mathrm{V}\end{array}$ & $\begin{array}{l}6 \\
4 . \\
5 \\
8\end{array}$ & II & $\begin{array}{l}6 \\
6 . \\
8 \\
4\end{array}$ & II & $\begin{array}{l}6 \\
9 . \\
1 \\
1\end{array}$ & II & $\begin{array}{l}7 \\
7 . \\
8 \\
8\end{array}$ & $\begin{array}{l}\text { II } \\
\text { I }\end{array}$ \\
\hline $\begin{array}{l}\text { Heilo } \\
\text { ngjia } \\
\text { ng }\end{array}$ & $\begin{array}{l}7 \\
4 . \\
9\end{array}$ & $\begin{array}{l}\text { II } \\
\text { I }\end{array}$ & $\begin{array}{l}88 \\
.5 \\
6\end{array}$ & $\begin{array}{l}\mathrm{I} \\
\mathrm{V}\end{array}$ & $\begin{array}{l}6 \\
0 . \\
9 \\
4 \\
\end{array}$ & II & $\begin{array}{l}6 \\
9 . \\
2 \\
9 \\
\end{array}$ & II & $\begin{array}{l}6 \\
7 . \\
5 \\
6 \\
\end{array}$ & II & $\begin{array}{l}7 \\
8 . \\
3 \\
3 \\
\end{array}$ & $\begin{array}{l}\text { II } \\
\text { I }\end{array}$ \\
\hline $\begin{array}{l}\text { Anhu } \\
\text { i }\end{array}$ & $\begin{array}{l}6 \\
2 . \\
4 \\
1 \\
\end{array}$ & II & 94 & $\begin{array}{l}\mathrm{I} \\
\mathrm{V}\end{array}$ & $\begin{array}{l}5 \\
9 . \\
9 \\
8 \\
\end{array}$ & II & $\begin{array}{l}7 \\
0 . \\
3 \\
8 \\
\end{array}$ & III & $\begin{array}{l}6 \\
1 . \\
1 \\
8 \\
\end{array}$ & II & $\begin{array}{l}8 \\
1 . \\
3 \\
4 \\
\end{array}$ & $\begin{array}{l}\text { II } \\
\text { I }\end{array}$ \\
\hline $\begin{array}{l}\text { Jiang } \\
\text { xi }\end{array}$ & $\begin{array}{l}7 \\
3 . \\
3 \\
3 \\
\end{array}$ & \begin{tabular}{|l|} 
II \\
I
\end{tabular} & $\begin{array}{l}95 \\
.5\end{array}$ & $\begin{array}{l}\mathrm{I} \\
\mathrm{V}\end{array}$ & $\begin{array}{l}6 \\
5 . \\
6 \\
2 \\
\end{array}$ & II & $\begin{array}{l}7 \\
4 . \\
8 \\
1 \\
\end{array}$ & III & $\begin{array}{l}6 \\
9 . \\
3 \\
7 \\
\end{array}$ & II & $\begin{array}{l}8 \\
4 . \\
5 \\
3 \\
\end{array}$ & $\begin{array}{l}\text { II } \\
\text { I }\end{array}$ \\
\hline $\begin{array}{l}\text { Hena } \\
\mathrm{n}\end{array}$ & $\begin{array}{l}7 \\
7 . \\
0 \\
9 \\
\end{array}$ & $\begin{array}{l}\text { II } \\
\text { I }\end{array}$ & $\begin{array}{l}92 \\
.2\end{array}$ & $\begin{array}{l}\text { I } \\
\text { V }\end{array}$ & $\begin{array}{l}5 \\
7 . \\
9 \\
5\end{array}$ & II & $\begin{array}{l}6 \\
7 . \\
1 \\
7 \\
\end{array}$ & II & $\begin{array}{l}6 \\
6 . \\
8 \\
4 \\
\end{array}$ & II & $\begin{array}{l}7 \\
8 . \\
7\end{array}$ & $\begin{array}{l}\text { II } \\
\text { I }\end{array}$ \\
\hline $\begin{array}{l}\text { Hube } \\
\mathrm{i}\end{array}$ & $\begin{array}{l}7 \\
5 . \\
8\end{array}$ & $\begin{array}{l}\text { II } \\
\text { I }\end{array}$ & $\begin{array}{l}92 \\
.1 \\
4\end{array}$ & $\begin{array}{l}\text { I } \\
\text { V }\end{array}$ & $\begin{array}{l}7 \\
2 . \\
2 \\
5 \\
\end{array}$ & $\begin{array}{l}\text { II } \\
\text { I }\end{array}$ & $\begin{array}{l}7 \\
8 . \\
4 \\
3 \\
\end{array}$ & III & $\begin{array}{l}7 \\
3 . \\
6 \\
5 \\
\end{array}$ & $\begin{array}{l}\text { II } \\
\text { I }\end{array}$ & $\begin{array}{l}8 \\
5 . \\
0 \\
1 \\
\end{array}$ & $\begin{array}{l}\text { I } \\
\text { V }\end{array}$ \\
\hline $\begin{array}{l}\text { Huna } \\
\mathrm{n}\end{array}$ & $\begin{array}{l}7 \\
0 . \\
9 \\
6 \\
\end{array}$ & \begin{tabular}{|l} 
II \\
I
\end{tabular} & $\begin{array}{l}93 \\
.0 \\
6\end{array}$ & $\begin{array}{l}\mathrm{I} \\
\mathrm{V}\end{array}$ & $\begin{array}{l}7 \\
0 . \\
9 \\
8\end{array}$ & $\begin{array}{l}\text { II } \\
\text { I }\end{array}$ & $\begin{array}{l}7 \\
6 . \\
2 \\
3\end{array}$ & III & $\begin{array}{l}7 \\
4 . \\
3 \\
9\end{array}$ & $\begin{array}{l}\text { II } \\
\text { I }\end{array}$ & $\begin{array}{l}8 \\
4 . \\
2 \\
2\end{array}$ & $\begin{array}{l}\text { II } \\
\text { I }\end{array}$ \\
\hline
\end{tabular}

Data source: according to the "China Energy Statistics Yearbook", "China Industrial Economic Statistical Yearbook", "China Statistical Yearbook", "Chinese economic census yearbook", "Regional Economic Statistical Yearbook".

\section{Empirical Analysis on the Influence of FDI on the Spatial and Temporal Differentiation of the Maturity of Energy Saving and Carbon Emission Reduction in Manufacturing Industry}

\subsection{Choice of Empirical Analysis Methods}

Because the influencing factors of spatial and temporal variation of the maturity of energy saving and carbon emission reduction in manufacturing industry have a certain fuzzy rules and the unity of certainty and uncertainty characteristics, this paper adopts the set pair analysis method based on fuzzy rules to verify the rationality of the influencing factor (FDI).

Set pair analysis requires the integration of the relevant information. Set pair and link degree $\left({ }^{\mu}\right.$ ) revealing the logical and dialectical relation between set pairs are two core concepts of this method.

$\mu_{i}=\frac{S}{N}+\frac{F_{1}}{N} i_{1}+\frac{F_{2}}{N} i_{2}+\frac{F_{3}}{N} i_{3}+\frac{P}{N} j$ $\mu=a+b_{1} i_{1}+b_{2} i_{2}+b_{3} i_{3}+c j$

( $\mu$ is a link degree of a certain level index) 6-unary link degree

$$
\mu=a+b_{1} i_{1}+b_{2} i_{2}+b_{3} i_{3}+b_{4} i_{4}+c j
$$

The main steps of the set pair analysis method based on fuzzy rule extraction:

Step 1: standardize $y_{G} n$ group $x_{i}$ data $y_{G 1} 、 x_{i 1} ; y_{G 2}$, $x_{i 2} ; \ldots y_{\sqsupset} 、 x_{i t} ; y_{G n} 、 x_{\sqcup}$ 。For qualitative data, see attached list 1.

$$
\begin{gathered}
\tilde{y}_{G t}=\frac{y_{G t}}{\max y_{G t}} \\
\tilde{x}_{i t}=\frac{x_{i t}}{\max x_{i t}}
\end{gathered}
$$

Step 2: calculate the degree of identity between ${ }^{\tilde{x}_{1 t}}, \tilde{x}_{2 t}, \tilde{x}_{3 t}, \ldots$ and $\tilde{y}_{G t}$ :

$a_{i t}=\frac{\min \left(\tilde{x}_{i t}, \tilde{y}_{G t}\right)}{\max \left(\tilde{x}_{i t}, \tilde{y}_{G t}\right)}$

Step 3: make a reasonable qualitative analysis of each ${ }^{a_{i t}}$, see attached list 1.

Step 4: coordination judge of $n a_{i t}$ at micro-level, see attached list 2.

Step 5: fuzzy rules extraction at macro-level. To obtain the mean value for tidentity degree $a_{i t}$, the greater the mean value of identity degree, the more significant the effect is.

$\overline{a_{i t}}=\frac{1}{p} \sum_{t=1}^{p} a_{i t}$

\subsection{Data Collection and Statistical Analysis}

Since the data in 2003 and 2012 are relatively centralized, the volatility and dispersion are low, the data in 2003 and 2012 are chosen as representative to be analyzed.

Data collection and statistical analysis of independent variable FDI: Table 2 is the situation of total foreign investment for the 11 cities in eastern China and 8 cities in Central China. In table 2, foreign investments in 2012 are higher than those in 2003, Chinese Eastern foreign investments are generally higher than those in central region. There is an imbalance of FDI in the eastern and central regions from time and space, reflecting the foreign direct investment has a spatial and temporal variation in

\begin{tabular}{|c|c|c|c|}
\hline \multirow{2}{*}{ regions } & \multirow{2}{*}{ provinces } & \multicolumn{2}{|c|}{$\begin{array}{l}\text { Total investment (billion dollars } \\
\text { ) }\end{array}$} \\
\hline & & 2003 & 2012 \\
\hline \multirow{11}{*}{$\begin{array}{l}\text { Eastern } \\
\text { region }\end{array}$} & Beijing & 463.28 & 1494 \\
\hline & Tianjin & 415.55 & 1189 \\
\hline & Hebei & 175.19 & 490 \\
\hline & Liaoning & 735.01 & 1856 \\
\hline & Shanghai & 1508.16 & 4138 \\
\hline & Jiangsu & 1500.14 & 6250 \\
\hline & Zhejiang & 612.26 & 2178 \\
\hline & Fujian & 661.18 & 1457 \\
\hline & Shandong & 596.62 & 1581 \\
\hline & Guangdong & 2412.62 & 4786 \\
\hline & Hainan & 89 & 271 \\
\hline \multirow{4}{*}{$\begin{array}{l}\text { Central } \\
\text { region }\end{array}$} & Shanxi & 61.19 & 320 \\
\hline & Jilin & 182.65 & 239 \\
\hline & Heilongjiang & 81.23 & 222 \\
\hline & Anhui & 116.44 & 400 \\
\hline
\end{tabular}
manufacturing industry.

\section{Table 2 Summary Sheet of Manufacturing FDI Data in 2003 and 2012}




\begin{tabular}{|l|l|l|}
\hline Jiangxi & 136.67 & 539 \\
\hline Henan & 126.28 & 463 \\
\hline Hubei & 176.76 & 583 \\
\hline Hunan & 126.28 & 384 \\
\hline
\end{tabular}

Data source: "China Statistical Yearbook"

The data collection and statistical analysis of dependent variables maturity: including the development index, coordination index and coordinated development index of the maturity of energy saving and carbon emission reduction in manufacturing industry, see table 1

\subsection{Empirical Analysis Process}

According to the principle, procedure and calculation formula of the set pair analysis method based on fuzzy rule extraction, combining the data in table 1 and table 2, the following table 3 is calculated. In table 3, in 2003, there are 7 cities in eastern and central regions with a highly collaborative identity degree between the coordinated development index and FDI. Among them, the eastern region has 7 cities, none is in the central region. There are 6 cities in eastern and central regions with a basic collaborative identity degree between the coordinated development index and FDI, of which there are 4 in the eastern region and 2 in the central region. In 2012, there are 14 cities in eastern and central regions with a highly collaborative identity degree between the coordinated development index and FDI, of which there are 10 in the eastern region and 4 in the central region. There are 5 cities in eastern and central regions with a basic collaborative identity degree between the coordinated development index and FDI, of which there are 1 in the eastern region and 4 in the central region.

From the longitudinal comparison, in 2012, the identity degree states between the coordinated development index and FDI in eastern and central regions are only two: highly coordinated and basic coordinated states. While in 2003, the identity degree states between the coordinated development index and FDI in eastern and central regions are four: highly coordinated state, basic coordinated state, slightly coordinated state and still coordinated state. Meanwhile, from the horizontal comparison, in 2003 and 2012, there are 7 cities in the eastern region with a highly collaborative identity degree between the coordinated development index and FDI, and none is in the central region.

Table 3 Data of FDI, Coordinated Development Index and Their Identity Degree in 2003 and 2012

\begin{tabular}{|c|c|c|c|c|c|c|c|}
\hline $\begin{array}{l}\mathrm{r} \\
\mathrm{e} \\
\mathrm{g}\end{array}$ & $\begin{array}{l}\mathrm{pr} \\
\mathrm{ov}\end{array}$ & \multicolumn{2}{|l|}{ FDI } & \multicolumn{2}{|c|}{$\begin{array}{l}\text { coordinated } \\
\text { development } \\
\text { index }\end{array}$} & \multicolumn{2}{|c|}{ identity degree } \\
\hline $\begin{array}{l}\mathrm{o} \\
\mathrm{n} \\
\mathrm{s}\end{array}$ & $\begin{array}{l}\text { ce } \\
\mathrm{s}\end{array}$ & $\begin{array}{l}200 \\
3\end{array}$ & $\begin{array}{l}201 \\
2\end{array}$ & 2003 & 2012 & 2003 & 2012 \\
\hline \multirow{3}{*}{$\begin{array}{l}\mathrm{E} \\
\mathrm{a} \\
\mathrm{s} \\
\mathrm{t} \\
\mathrm{e} \\
\mathrm{r} \\
\mathrm{n} \\
\mathrm{r} \\
\mathrm{e}\end{array}$} & $\begin{array}{l}\text { Be } \\
\text { iji } \\
\text { ng }\end{array}$ & $\begin{array}{l}0.19 \\
202 \\
360 \\
9\end{array}$ & $\begin{array}{l}0.2 \\
390 \\
4\end{array}$ & $\begin{array}{l}0.9281 \\
69 \\
\quad \text { (high }\end{array}$ & 1 (high & $\begin{array}{l}0.20688 \\
4 \\
\text { highly } \\
\text { coordina } \\
\text { ted }\end{array}$ & $\begin{array}{l}0.23904 \\
\text { highly } \\
\text { coordinated }\end{array}$ \\
\hline & $\begin{array}{l}\mathrm{Ti} \\
\text { an } \\
\text { jin }\end{array}$ & $\begin{array}{l}0.17 \\
224 \\
013 \\
7\end{array}$ & $\begin{array}{l}0.1 \\
902 \\
4\end{array}$ & $\begin{array}{l}0.9685 \\
39 \\
\quad \text { (high } \\
\text { ) }\end{array}$ & $\begin{array}{l}0.9098 \\
86 \\
\quad \text { (high } \\
\text { ) }\end{array}$ & $\begin{array}{l}0.17783 \\
5 \\
\text { highly } \\
\text { coordina } \\
\text { ted } \\
\end{array}$ & $\begin{array}{l}0.209081 \\
\text { highly } \\
\text { coordinated }\end{array}$ \\
\hline & $\begin{array}{l}\mathrm{He} \\
\text { be } \\
\text { i }\end{array}$ & $\begin{array}{l}0.07 \\
261 \\
400 \\
5\end{array}$ & $\begin{array}{l}0.0 \\
784\end{array}$ & $\begin{array}{l}0.6970 \\
00 \\
\text { (mediu } \\
\text { m high) }\end{array}$ & $\begin{array}{l}0.8315 \\
40 \\
\quad \text { (on } \\
\text { the } \\
\text { high } \\
\text { side) } \\
\end{array}$ & $\begin{array}{l}0.10418 \\
1 \\
\text { still } \\
\text { coordina } \\
\text { ted }\end{array}$ & $\begin{array}{l}0.094283 \\
\text { basic } \\
\text { coordinated }\end{array}$ \\
\hline $\begin{array}{l}\mathrm{g} \\
\mathrm{i} \\
\mathrm{o} \\
\mathrm{n}\end{array}$ & $\begin{array}{l}\mathrm{Li} \\
\text { ao } \\
\mathrm{ni} \\
\mathrm{ng}\end{array}$ & $\begin{array}{l}0.30 \\
465 \\
220 \\
4\end{array}$ & $\begin{array}{l}0.2 \\
969 \\
6\end{array}$ & $\begin{array}{l}0.7859 \\
72 \\
\text { slightly } \\
\text { higher } \\
\text { ) }\end{array}$ & $\begin{array}{l}0.9093 \\
25 \\
\text { (high }\end{array}$ & $\begin{array}{l}0.38761 \\
2 \\
\text { slightly } \\
\text { coordina } \\
\text { ted }\end{array}$ & $\begin{array}{l}0.326572 \\
\text { highly } \\
\text { coordinated }\end{array}$ \\
\hline & $\begin{array}{l}\text { Sh } \\
\text { an } \\
\text { gh } \\
\text { ai }\end{array}$ & $\begin{array}{l}0.62 \\
511 \\
294 \\
8\end{array}$ & $\begin{array}{l}0.6 \\
620 \\
8\end{array}$ & $\begin{array}{l}0.9506 \\
09 \\
\quad \text { (high } \\
\text { ) }\end{array}$ & $\begin{array}{l}0.9512 \\
44 \\
\quad \text { (high } \\
\text { ) }\end{array}$ & $\begin{array}{l}0.65759 \\
2 \\
\text { highly } \\
\text { coordina } \\
\text { ted }\end{array}$ & $\begin{array}{l}0.696015 \\
\text { highly } \\
\text { coordinated }\end{array}$ \\
\hline
\end{tabular}

\begin{tabular}{|c|c|c|c|c|c|c|}
\hline $\begin{array}{l}\text { Jia } \\
\text { ng } \\
\text { su }\end{array}$ & $\begin{array}{l}0.62 \\
178 \\
876 \\
1\end{array}$ & 1 & $\begin{array}{l}0.8990 \\
76 \\
\text { (on } \\
\text { the } \\
\text { high } \\
\text { side) }\end{array}$ & $\begin{array}{l}0.9151 \\
54 \\
\text { (high }\end{array}$ & $\begin{array}{l}0.69158 \\
7 \\
\text { basic } \\
\text { coordina } \\
\text { ted }\end{array}$ & $\begin{array}{l}0.915154 \\
\text { highly } \\
\text { coordinated }\end{array}$ \\
\hline $\begin{array}{l}\text { Zh } \\
\text { eji } \\
\text { an } \\
\text { g }\end{array}$ & $\begin{array}{l}0.25 \\
377 \\
390 \\
6\end{array}$ & $\begin{array}{l}0.3 \\
484 \\
8\end{array}$ & $\begin{array}{l}0.9477 \\
90 \\
\text { (high }\end{array}$ & $\begin{array}{l}0.9227 \\
753 \\
\text { (high } \\
\text { (high }\end{array}$ & $\begin{array}{l}0.26775 \\
3 \\
\text { highly } \\
\text { coordina } \\
\text { ted }\end{array}$ & $\begin{array}{l}0.377643 \\
\text { highly } \\
\text { coordinated }\end{array}$ \\
\hline $\begin{array}{l}\text { Fu } \\
\text { jia } \\
\text { n }\end{array}$ & $\begin{array}{l}0.27 \\
405 \\
061 \\
7\end{array}$ & $\begin{array}{l}0.2 \\
331 \\
2\end{array}$ & $\begin{array}{l}0.9462 \\
11 \\
\text { (high }\end{array}$ & $\begin{array}{l}0.9498 \\
99 \\
\text { (high }\end{array}$ & $\begin{array}{l}0.28969 \\
\text { highly } \\
\text { coordina } \\
\text { ted }\end{array}$ & $\begin{array}{l}0.245416 \\
\text { highly } \\
\text { coordinated }\end{array}$ \\
\hline $\begin{array}{l}\text { Sh } \\
\text { an } \\
\text { do } \\
\text { ng }\end{array}$ & $\begin{array}{l}0.24 \\
729 \\
132 \\
6\end{array}$ & $\begin{array}{l}0.2 \\
529 \\
6\end{array}$ & $\begin{array}{l}0.8323 \\
18 \\
\text { (on } \\
\text { the } \\
\text { high } \\
\text { side) }\end{array}$ & $\begin{array}{l}0.9079 \\
80 \\
\text { (high }\end{array}$ & $\begin{array}{l}0.29711 \\
1 \\
\text { basic } \\
\text { coordina } \\
\text { ted }\end{array}$ & $\begin{array}{l}0.278596 \\
\text { highly } \\
\text { coordinated }\end{array}$ \\
\hline $\begin{array}{l}\mathrm{Gu} \\
\text { an } \\
\text { gd } \\
\text { on } \\
\text { g }\end{array}$ & 1 & $\begin{array}{l}0.7 \\
657 \\
6\end{array}$ & ${ }^{1}$ (high & $\begin{array}{l}0.9544 \\
95 \\
\text { (high } \\
\text { ) }\end{array}$ & $\begin{array}{l}1 \\
\text { highly } \\
\text { coordina } \\
\text { ted }\end{array}$ & $\begin{array}{l}0.802268 \\
\text { highly } \\
\text { coordinated }\end{array}$ \\
\hline $\begin{array}{l}\text { Ha } \\
\text { in } \\
\text { an }\end{array}$ & $\begin{array}{l}0.03 \\
688 \\
935 \\
7\end{array}$ & $\begin{array}{l}0.0 \\
433 \\
6\end{array}$ & $\begin{array}{l}0.9131 \\
70 \\
\text { (high }\end{array}$ & $\begin{array}{l}0.9228 \\
87 \\
\text { (high }\end{array}$ & $\begin{array}{l}0.04039 \\
7 \\
\text { highly } \\
\text { coordina } \\
\text { ted }\end{array}$ & $\begin{array}{l}0.046983 \\
\text { highly } \\
\text { coordinated }\end{array}$ \\
\hline $\begin{array}{l}\text { Sh } \\
\text { an } \\
\text { xi }\end{array}$ & $\begin{array}{l}0.02 \\
536 \\
246 \\
9\end{array}$ & $\begin{array}{l}0.0 \\
512\end{array}$ & $\begin{array}{l}0.7729 \\
59 \\
( \\
\text { slightly } \\
\text { high) }\end{array}$ & $\begin{array}{l}0.8206 \\
68 \\
\text { (on } \\
\text { the } \\
\text { high } \\
\text { side) }\end{array}$ & $\begin{array}{l}0.04426 \\
6 \\
\text { slightly } \\
\text { coordina } \\
\text { ted }\end{array}$ & $\begin{array}{l}18.53956 \\
\text { basic } \\
\text { coordinated }\end{array}$ \\
\hline $\begin{array}{l}\text { Jili } \\
\text { n }\end{array}$ & $\begin{array}{l}0.07 \\
570 \\
607 \\
9\end{array}$ & $\begin{array}{l}0.0 \\
382 \\
4\end{array}$ & $\begin{array}{l}0.7793 \\
1 \\
\text { ( } \\
\text { slightly } \\
\text { higher } \\
\text { ) }\end{array}$ & $\begin{array}{l}0.8728 \\
98 \\
\text { (on } \\
\text { the } \\
\text { high } \\
\text { side) }\end{array}$ & $\begin{array}{l}0.09714 \\
4 \\
\text { slightly } \\
\text { coordina } \\
\text { ted }\end{array}$ & $\begin{array}{l}8.985623 \\
\text { basic } \\
\text { coordinated }\end{array}$ \\
\hline $\begin{array}{l}\mathrm{He} \\
\text { ilo } \\
\text { ng } \\
\text { jia } \\
\text { ng }\end{array}$ & $\begin{array}{l}0.03 \\
366 \\
879 \\
2\end{array}$ & $\begin{array}{l}0.0 \\
355 \\
2\end{array}$ & $\begin{array}{l}0.7618 \\
40 \\
( \\
\text { slightly } \\
\text { higher } \\
\text { ) }\end{array}$ & $\begin{array}{l}0.8779 \\
42 \\
\text { (on } \\
\text { the } \\
\text { high } \\
\text { side) }\end{array}$ & $\begin{array}{l}0.04419 \\
4 \\
\text { slightly } \\
\text { coordina } \\
\text { ted }\end{array}$ & $\begin{array}{l}19.86563 \\
\text { basic } \\
\text { coordinated }\end{array}$ \\
\hline $\begin{array}{l}\text { An } \\
\text { hu } \\
\text { i }\end{array}$ & $\begin{array}{l}0.04 \\
826 \\
288 \\
4\end{array}$ & $\begin{array}{l}0.0 \\
64\end{array}$ & $\begin{array}{l}0.6898 \\
96 \\
\text { (mediu } \\
\text { m high) } \\
\end{array}$ & $\begin{array}{l}0.9116 \\
79 \\
\text { (high }\end{array}$ & $\begin{array}{l}0.06995 \\
7 \\
\text { still } \\
\text { coordina } \\
\text { ted }\end{array}$ & $\begin{array}{l}13.03204 \\
\text { highly } \\
\text { coordinated }\end{array}$ \\
\hline $\begin{array}{l}\text { Jia } \\
\text { ng } \\
\text { xi }\end{array}$ & $\begin{array}{l}0.05 \\
664 \\
795 \\
9\end{array}$ & $\begin{array}{l}0.0 \\
862 \\
4\end{array}$ & $\begin{array}{l}0.7822 \\
51 \\
( \\
\text { slightly } \\
\text { higher } \\
\text { ) }\end{array}$ & $\begin{array}{l}0.9474 \\
33 \\
\text { (high }\end{array}$ & $\begin{array}{l}0.07241 \\
7 \\
\text { slightly } \\
\text { coordina } \\
\text { ted }\end{array}$ & $\begin{array}{l}13.08309 \\
\text { highly } \\
\text { coordinated }\end{array}$ \\
\hline $\begin{array}{l}\mathrm{He} \\
\text { na } \\
\mathrm{n}\end{array}$ & $\begin{array}{l}0.05 \\
234 \\
143 \\
8\end{array}$ & $\begin{array}{l}0.0 \\
740 \\
8\end{array}$ & $\begin{array}{l}0.7537 \\
21 \\
( \\
\text { slightly } \\
\text { higher } \\
\text { ) }\end{array}$ & $\begin{array}{l}0.8820 \\
89 \\
\text { (on } \\
\text { the } \\
\text { high } \\
\text { side) }\end{array}$ & $\begin{array}{l}0.06944 \\
4 \\
\text { slightly } \\
\text { coordina } \\
\text { ted }\end{array}$ & $\begin{array}{l}12.70216 \\
\text { basic } \\
\text { coordinated }\end{array}$ \\
\hline $\begin{array}{l}\mathrm{H} \\
\mathrm{ub} \\
\mathrm{ei}\end{array}$ & $\begin{array}{l}0.07 \\
326 \\
475\end{array}$ & $\begin{array}{l}0.0 \\
932 \\
8\end{array}$ & $\begin{array}{l}0.8305 \\
14 \\
\text { (on } \\
\text { the } \\
\text { high } \\
\text { side) }\end{array}$ & $\begin{array}{l}0.9528 \\
13 \\
\text { (high }\end{array}$ & $\begin{array}{l}0.08821 \\
6 \\
\text { basic } \\
\text { coordina } \\
\text { ted }\end{array}$ & $\begin{array}{l}10.8009 \\
\text { highly } \\
\text { coordinated }\end{array}$ \\
\hline $\begin{array}{l}\mathrm{H} \\
\text { un } \\
\text { an }\end{array}$ & $\begin{array}{l}0.05 \\
234 \\
143 \\
8\end{array}$ & $\begin{array}{l}0.0 \\
614 \\
4\end{array}$ & $\begin{array}{l}0.8388 \\
59 \\
\text { (on } \\
\text { the } \\
\text { high } \\
\text { side) }\end{array}$ & $\begin{array}{l}0.9439 \\
59 \\
\text { (high }\end{array}$ & $\begin{array}{l}0.06239 \\
6 \\
\text { basic } \\
\text { coordina } \\
\text { ted }\end{array}$ & $\begin{array}{l}15.12851 \\
\text { highly } \\
\text { coordinated }\end{array}$ \\
\hline
\end{tabular}


Data source: "China Statistical Yearbook"

\subsection{Empirical Analysis Results}

From the analysis in table 3.2, referring to the identity degree between the coordinated development index and FDI, there are spatial and temporal differentiation characteristics for the influence of FDI on the maturity of energy saving and carbon emission reduction in manufacturing industry. In terms of time, the impact of FDI on the maturity of energy saving and carbon emission reduction in manufacturing industry in 2012 was higher than that in 2003 in Chinese central and eastern regions. In terms of space, the impact of FDI in the eastern region on the maturity of energy saving and carbon emission reduction in the manufacturing sector is significantly higher than that in the central region.

\section{Suggestions on Improving the Maturity of Energy Saving and} Carbon Emission Reduction in Manufacturing Industry

\subsection{Improve the Quality of Attracting Foreign Investment}

In attracting foreign investment, manufacturing enterprises should pay attention to the quality of investment, and the introduction of foreign enterprises with advanced technology of energy saving and carbon emission reduction. Before the introduction, manufacturing enterprises should do a series of comparative analysis about their investment capacity, their technology of energy saving and carbon emission reduction and their energy consumption degree and so on. Detailed situation of energy saving and carbon emission reduction of foreign enterprises should be understood, so as to improve the positive effect of FDI on the maturity of energy saving and carbon emission reduction in manufacturing industry.

\subsection{Strengthen the Technological Innovation Capability of Manufacturing Enterprises}

As the main body of technological innovation, manufacturing enterprises should establish a development system and an effective operational mechanism of energy saving and carbon emission reduction to meet the need of market competition and enterprise development, so as to improve the response ability of manufacturing enterprise to the market, the capacity of resources utilization and the capability of independent innovation on energy saving and carbon emission reduction.

\subsection{Strengthen Cross- regional and International Cooperation}

The regional cooperation in central and eastern regions should be strengthen to form the state that advanced technologies of energy saving and carbon emission reduction in the eastern region will promote the development of that in the central region, thereby enhancing the mature development of energy saving and carbon emission reduction of the entire manufacturing industry. At the same time, the cooperation among the countries all over the world should be strengthened, and the advanced technologies of developed countries should be used to drive China's technologies of energy saving and carbon emission reduction.

\subsection{Improve the Relevant Laws and Regulations and System}

China's economy has made great achievements, but it should not be at the expense of the environment. Therefore, the government should improve the relevant laws and regulations on energy conservation and carbon emission reduction, implement the most stringent environmental protection law, and restrict activities of energy saving and carbon emission reduction in manufacturing enterprises through laws and regulations, so as to enhance the capacity of energy saving and carbon emission reduction. At last, corresponding checks on energy saving and carbon emission reduction should be carried out at regular intervals to promote the maturity of energy saving and carbon emission reduction of the entire manufacturing industry.

\section{References}

[1] Wang Wenju, Xiang Feng. Evaluation of China's industrial structure and energy saving and emission reduction potential[J]. China industrial economy, 2015, (1): 44-56.

[2] Ren Lili, Nie Ming. Study on the difference of K efficiency in China's industrial carbon emissions based on nonparametric frontier analysis [J]. Quantitative economic, technical and economic research, 2015, (9): 58-70.
[3] LAN Qinqin. FDI on Chinese manufacturing[D]. Study on the effect of industry agglomeration in Hunan: Xiangtan University; master's thesis, 2015.

[4] Fan Dingxiang, Liu Huihong. Analysis of the distribution characteristics and changes of carbon emission intensity in China's provinces[J]. Journal of Xi'an Institute of Finance and Economics, 2015,25 (5): 5 -10.

[5] Li Zihao, Liu Huihuang. The effect of technical effects of FDI on carbon emissions[J]. China population, resources and environment,.2014 (12): 610.

[6] Niu Haixia, Hu Jiayu. FDI trade and CO2 emission in China An Empirical Study on the correlation between [J]. 2014341 (5): 100 - 109.

[7] Wang Qunwei, Zhou Peng, Zhou Dequn. Dynamic changes, regional differences and influencing factors of carbon dioxide emission performance in China[J]. Chinese population, resources and environment, 2010, (1): 45-108.

[8] Tang Lamei. FDI of China's manufacturing industry, and empirical research on environmental pollution and environmental regulation of Hunan[D]. Hunan University doctoral dissertation, 2014.;

[9] Yang Xiaoming, Tian Peng. Factors of FDI location choice of the three economic circles of China and the central and western regions of the empirical research on[J]. Financial research, 2014, (11): 98-107.

[10] Zhang Yinghua, Wang Dandan. Situation analysis and countermeasure suggestion of low carbon development in manufacturing industry [J]. Macroeconomic management, 2013 (1): 45-101.

\section{Attached lists}

Schedule 1 qualitative language list

\begin{tabular}{|c|c|c|c|}
\hline rade ${ }^{G}$ & $\begin{array}{l}\text { normaliz } \\
\text { ation data } \\
\text { or } \\
\text { identity } \\
\text { degree }\end{array}$ & $\begin{array}{l}\text { qualitative } \\
\text { normalization data }\end{array}$ & $\begin{array}{l}\text { qualitative identity } \\
\text { degree }\end{array}$ \\
\hline$\overline{1}$ & $0.9-1.0$ & high & highly coordinated \\
\hline 2 & $0.8-0.9$ & on the high side & basic coordinated \\
\hline 3 & $0.7-0.8$ & slightly higher & slightly coordinated \\
\hline 4 & $0.6-0.7$ & medium high & still coordinated \\
\hline 5 & $0.5-0.6$ & medium & $\begin{array}{l}\text { criticality } \\
\text { coordinated }\end{array}$ \\
\hline 6 & $0.4-0.5$ & medium low & $\begin{array}{l}\text { criticality of non- } \\
\text { coordination }\end{array}$ \\
\hline 7 & $0.3-0.4$ & on the low side & $\begin{array}{l}\text { on the side of non- } \\
\text { coordination }\end{array}$ \\
\hline 8 & $0.2-0.3$ & low & $\begin{array}{ll}\text { not } & \text { cooperative } \\
\text { enough } & \end{array}$ \\
\hline 9 & $0.1-0.2$ & very low & very uncoordinated \\
\hline 10 & $0-0.1$ & extremely low & $\begin{array}{l}\text { extremely } \\
\text { uncoordinated }\end{array}$ \\
\hline
\end{tabular}

Schedule 2 coordination decision sheet

\begin{tabular}{|l|l|}
\hline nge ra & qualitative coordination \\
\hline 0 & identical (fully coordinated) \\
\hline $1-2$ & on the side of coordination \\
\hline $3-4$ & slightly identical (slightly coordinated) \\
\hline $5-6$ & $\begin{array}{l}\text { critical deviation (coordination at critical and on the side of } \\
\text { non-coordination) }\end{array}$ \\
\hline $7-8$ & deviation to the opposition( on the side of non-coordination) \\
\hline $9-10$ & $\begin{array}{l}\text { oppose (completely without coordination) and present as an } \\
\text { antagonistic state }\end{array}$ \\
\hline
\end{tabular}

8. About the Authors

The last item in the manuscript should be a brief biographical sketch of each author, typed in 10-point Times New Roman, single-spaced (However it is optional). 